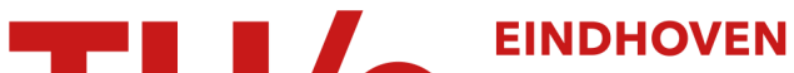 UNIVERSITY OF TECHNOLOGY
}

\section{Realisation of cost-informed process support within the YAWL workflow environment}

\section{Citation for published version (APA):}

Adams, M., Wynn, M. T., Ouyang, C., \& Hofstede, ter, A. H. M. (2015). Realisation of cost-informed process support within the YAWL workflow environment. In J. Bae, S. Suriadi, \& L. Wen (Eds.), Proceedings of the Third Asia Pacific Conference, AP-BPM 2015, June 24-26 2015, Busan, South Korea (pp. 3-18). (Lecture Notes in Business Information Processing; Vol. 219). Springer. https://doi.org/10.1007/978-3-319-19509-4_1

DOI:

10.1007/978-3-319-19509-4_1

Document status and date:

Published: 01/01/2015

\section{Document Version:}

Publisher's PDF, also known as Version of Record (includes final page, issue and volume numbers)

\section{Please check the document version of this publication:}

- A submitted manuscript is the version of the article upon submission and before peer-review. There can be important differences between the submitted version and the official published version of record. People interested in the research are advised to contact the author for the final version of the publication, or visit the $\mathrm{DOI}$ to the publisher's website.

- The final author version and the galley proof are versions of the publication after peer review.

- The final published version features the final layout of the paper including the volume, issue and page numbers.

Link to publication

\section{General rights}

Copyright and moral rights for the publications made accessible in the public portal are retained by the authors and/or other copyright owners and it is a condition of accessing publications that users recognise and abide by the legal requirements associated with these rights.

- Users may download and print one copy of any publication from the public portal for the purpose of private study or research.

- You may not further distribute the material or use it for any profit-making activity or commercial gain

- You may freely distribute the URL identifying the publication in the public portal.

If the publication is distributed under the terms of Article 25fa of the Dutch Copyright Act, indicated by the "Taverne" license above, please follow below link for the End User Agreement:

www.tue.nl/taverne

Take down policy

If you believe that this document breaches copyright please contact us at:

openaccess@tue.nl

providing details and we will investigate your claim. 


\title{
Realisation of Cost-Informed Process Support Within the YAWL Workflow Environment
}

\author{
M. Adams ${ }^{1(\bowtie)}$, M.T. Wynn ${ }^{1}$, C. Ouyang ${ }^{1}$, and A.H.M. ter Hofstede ${ }^{1,2}$ \\ ${ }^{1}$ Queensland University of Technology, Brisbane, Australia \\ $\{$ mj . adams, $m$. wynn, c . ouyang, a.terhof stede\}@qut. edu . au \\ 2 Eindhoven University of Technology, Eindhoven, The Netherlands
}

\begin{abstract}
Organisations are always focussed on ensuring that their business operations are performed in the most cost-effective manner, and that processes are responsive to ever-changing cost pressures. In many organisations, however, strategic cost-based decisions at the managerial level are not directly or quickly translatable to process-level operational support. A primary reason for this disconnect is the limited system-based support for cost-informed decisions at the process-operational level in real time. In this paper, we describe the different ways in which a workflow management system can support process-related decisions, guided by cost-informed considerations at the operational level, during execution. As a result, cost information is elevated from its non-functional attribute role to a first-class, fully functional process perspective. The paper defines success criteria that a WfMS should meet to provide such support, and discusses a reference implementation within the YAWL workflow environment that demonstrates how the various types of costinformed decision rules are supported, using an illustrative example.
\end{abstract}

Keywords: Cost-informed process enactment • Workflow management • Yet Another Workflow Language (YAWL)

\section{Introduction}

Organisations are constantly seeking cost efficiencies in their day-to-day operations, and are eager to have cost-based considerations applied to their business processes in a timely manner. In most organisations, however, there is a temporal disconnect between the making of strategic cost-based decisions and having them applied as business rules in automated business processes. Our observation is that most Workflow Management Systems (WfMSs) offer no support for cost considerations beyond the use of generic non-functional attributes or basic a posteriori reporting. Detailed real-time cost information is typically unavailable during process execution and, as a result, system-based decisions cannot be made within the context of a process instance while it is active, nor can it be used for live monitoring or other operational decision support.

Cost is often described as a non-functional requirement (NFR) for a software system, in the same class as requirements such as maintainability, usability,

(C) Springer International Publishing Switzerland 2015

J. Bae et al. (Eds.): AP-BPM 2015, LNBIP 219, pp. 3-18, 2015.

DOI: $10.1007 / 978-3-319-19509-4 \_1$ 
reliability, traceability, quality or safety [1]. However, care must be taken to distinguish between the implementation cost requirements of a host processaware system and the impact of a cost-informed perspective on each of the processes it subsequently executes and manages. A WfMS is a particular kind of software system that executes process models that in turn are very closely linked with cost implications at all scopes of a business process: individual activities, resources, process instances and execution sets. Also, the dynamic and sometimes ad-hoc nature of strategic cost-related decisions makes their timely application of utmost importance at both the design time and runtime phases of a process. There are potentially significant cost savings that an organisation can achieve through WfMS support for system-based, real-time, cost-informed decisions.

A short research paper presenting a conceptual framework that defines how WfMSs can provide sophisticated support for strategic cost-informed operational decisions was published in [2]. This paper extends the earlier work by providing a detailed discussion of the realisation of the cost-informed operational support within the well-known open-source WfMS system environment YAWL [3], illustrated with a familiar example. The remainder of the paper is organised as follows. Section 2 provides a review of the conceptual framework. Section 3 defines the success criteria that need to be satisfied before a WfMS can be considered cost-informed. Section 4 discusses the implementation of a Cost Service within YAWL. Section 5 describes an example that illustrates the implementation. Section 6 presents related work and Sect. 7 concludes the paper.

\section{A Framework for Cost-Informed Decisions}

In order for WfMSs to support cost-informed decision making during process execution, a number of key requirements must be fulfilled. Firstly, a WfMS must be 'cost-aware', i.e. there must be support for the ability to link cost information (e.g. cost rates, cost functions) to different elements in a process (e.g. activities, case attributes and durations) and to resources (e.g. names, roles or experience levels). Secondly, a cost-informed WfMS should provide the ability to specify cost-informed control flow definitions and resource allocation rules at design time and to provide support for system-based decisions and system-supported user decisions at runtime. Thirdly, a cost-informed WfMS should provide the ability to compute processing costs of a case at runtime and to provide a mechanism for a detailed cost analysis at post-execution time.

Figure 1 depicts a conceptual framework which describes (1) data input, i.e. the information requirements to enact actions that can be undertaken by or with a WfMS to support cost-informed decision making, (2) the actions that can be taken at the levels of process, activity, and resource (work distribution), and (3) the cost-informed support that is delivered, either through decisions by the WfMS itself or people using its support.

To support cost-informed actions with a WfMS, we require an executable process model and a cost model that describes all cost rates/data associated with activities within the process. Cost data could be as simple or as complex as an 


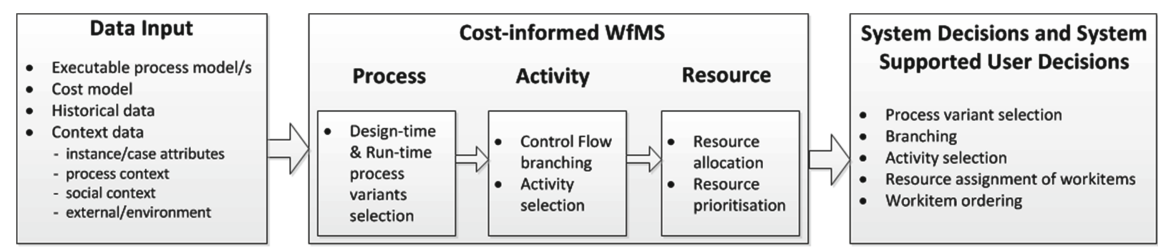

Fig. 1. A framework supporting cost-informed process execution with a WfMS.

organisation requires. For instance, it could be a variable cost that describes the hourly rate of a resource, but it could also be a function to compute the cost of an activity based on a combination of the resource assigned at runtime, the type of case being executed and the duration of the executed activity. Cost information, together with historical data as stored in a so-called process log regarding past executions, can be used to determine the cost of process executions, as illustrated in our earlier work [4]. Since a business process is always executed in a particular context, we also adopt the four levels of context data described in [5]: case attributes, process context, social context, and the environment.

All cost-informed actions supported by a WfMS are informed by data input and governed by strategic cost optimisation goals. These actions can be classified into three levels: process, activity, and resource.

Process. The process level is concerned with making cost-informed process selection decisions based on cost information of processes or process variants at design time or at runtime. This may involve the selection among different processes or selection among different process variants (which are created from individual processes during the execution phase). It should be possible to assign a (whole) process or process variant to a certain resource team for execution (i.e. outsourcing) based on the cost profile and a certain set of cost-based selection rules. For instance, a WfMS may make an automated selection of a process variant based on predefined cost-based selection rules. Alternatively, the WfMS may provide an administrator with cost profiles of different process variants and s/he can make an informed selection.

Activity. For cases that have been started under the control of a WMFS, it is necessary to decide at certain points about the activity (or activities) to be executed next. In its coordination capability, a WfMS may decide on which activity instances are enabled in a specific case, based on the branching conditions specified in the underlying process. As such, the WfMS can make a cost-informed decision based on a pre-defined business rule to enable/start an activity. In a similar manner, a WfMS could also skip, suspend or cancel activity instances, among other actions, based on that cost information. Or, an administrator can do so based on the cost information presented to them.

Resource. For activity instances that need to be carried out by a user, both "push" and "pull" patterns of activity-resource assignment [6] should be supported. That is, a WfMS would need to support cost-informed resource allocation rules for selecting a resource to offer, allocate, and/or start an activity 
instance and to support a user to make such cost-informed activity selection decisions. Finally, a WfMS should provide support for ordering and prioritisation of work based on cost information. That means that support for multiple activity instances to be ranked based on expected cost is required.

\section{Implementation Success Criteria}

For a WfMS to be capable of providing full cost-informed support across all three levels (process, activity and resource), it is essential that the following key success criteria be satisfied.

1. Associating Processes with Cost Data and Cost-based Rules. Processes, activities and resources must have their relevant cost rates specified, stored and accessible during execution. Example values would include salary and incidental costs for human resources, the costs of required materials and consumables, and fixed costs associated with activity enactments, rentals, depreciation, etc. Definitions of such costs would normally be acquired at process design time and either incorporated directly into the process specification or linked to it via an external file or database.

2. Dynamic Cost Calculations at Runtime. The dynamic runtime calculation of the cost of each process instance and its components is required so that real-time system- and human-based decisions can be applied. Such calculations would fall into the following categories:

- time-based, e.g. salary costs for the period a human resource spends on performing an activity, or timed charges for interaction with an external service, or the cost of insurance for the duration of an activity;

- usage-based, e.g. forklift hire, or the use of an MRI machine, or payment of a set fee for an expert witness;

- measurement-based, e.g. per tonne costs of a raw material, or per millilitre of a pharmaceutical, or per kilowatt-hour of an energy supply;

- invocation-based, e.g. costs involved in retooling an assembly line for a product run;

- a fixed cost, e.g. an overhead cost of commencing an activity, or a building approval application fee;

- a combination of the above, e.g. a truck rental may involve an initial hire cost plus a fee per kilometre plus a fuel cost, or a usage-based fee for a machine hire may also involve a time-based insurance fee.

3. Cost Data Logging and Analysis. All calculated costs for each process instance and component activities must be incorporated into the process event logs so that they can be reviewed by management during and post execution, and used to perform extrapolated calculations over archived data in real time as required for the next criterion.

4. Support for Cost-informed Decisions. A cost-informed WfMS must support the ability to use the calculated costs of the current process instance and its components, and/or those of all previous instances of the process, to: 
- make human-based and system-based cost-informed control-flow decisions. These decisions would include providing real time calculated values for use as input into branching predicates; to continuously monitor for cost overruns and, when detected, manually or dynamically skip unnecessary or low priority work items, or to cancel work items and/or cases; and to notify administrators when cost thresholds are being approached;

- allocate work to resources based on automated decisions about their costs;

- provide human resources and administrators with cost information about a process and its component activities, to enable them to make cost-informed decisions about subsequent process executions and process re-engineering;

- support for cost-informed process variant selections so that the most appropriate activities are selected and performed based on the current context of the process instance, the calculated costs associated with that context, and archival cost data relevant to the current process execution.

\section{Realisation}

We have developed an implementation for cost-informed support within the YAWL system environment [3] that fully addresses the success criteria outlined in the previous section. YAWL was chosen as the implementation platform because it is built on an expressive workflow language that provides extensive support for identified workflow and resource patterns. The environment is opensource and offers a service-oriented and vastly extensible architecture, allowing the prototype to be implemented completely independent of the core workflow (enactment) engine.

\subsection{The Cost Service}

A component service, known as the Cost Service, has been realised as a YAWL Custom Service [3]. Satisfying the success criteria using a discrete component provides a solution that remains orthogonal to the main process enactment engine, allowing the approach to be applicable to multiple environments.

The service provides three external REST-based interfaces:

- an evaluation interface, which receives notifications from the workflow engine and participating support services at various points in the runtime life-cycle of a process instance, and through which the engine and services may query cost-information, either to request a calculation and have the result returned or to evaluate a cost predicate to a boolean value. Such requests are handled by the predicate $\&$ expression evaluator sub-component of the service;

- a $\log$ interface, through which the workflow engine and participating support services may request a complete cost-annotated log of a process instance (or instances) via the log annotator sub-component; and

- a model interface, which supports the import and export of cost models. Such models are stored in the model handler sub-component. 
Cost Model. A cost model is an XML document that describes all the base cost data and formulae to be associated with a particular process model as defined in [4]. In brief, each cost model consists of three core descriptor sets:

- Drivers: Each cost driver defines how cost is associated with one or more process elements (resource, activity, case) together with the relevant cost rate for each element. A cost rate is defined as a data pair of a value and a per amount, for example $\$ 50$ per hour, $\$ 70$ per tonne, $\$ 20$ per invocation (fixed) and so on, applied to a process element. An entire process may have any number of drivers defined for it.

- Functions: Each function defines an expression for aggregating various cost elements. For example, a function may aggregate a fixed cost, and costs for salaries, insurance and machine hire for all resources involved in an activity.

- Mappings: Each mapping provides a method for relating terms used in management accounting to equivalent terms used in a WfMS.

All of the cost model definitions, and the runtime calculations based on them, are managed by the Cost Service. Each imported cost model is persisted by the Cost Service so that it is always available across future executions of the related process, and across server restarts.

The Cost Service is also responsible for the logging of all cost data for all process instances and their activities. The workflow engine and other interested services such as the Resource Service, which manages all resourcing allocations, notify the Cost Service throughout the life-cycle of each process instance, passing to it the appropriate data so that it can (i) perform the required cost calculations by applying the contextual data (regarding resources, activities, durations, and so on) to the relevant cost model components; and (ii) store all interactions and results in its process logs.

The result of each function call, in addition to being logged by the service, is also returned to the caller for its own decision making and/or logging purposes, as required. Other services may also interface with the Cost Service and have cost data logged with activity completion in the engine (via separately defined logging predicates).

Cost-Informed Predicates. The YAWL control-flow predicate syntax has been extended to include cost-informed expressions. A cost-informed predicate consists of a conditional expression that may be used to evaluate specified current or historical costs, a comparison operator and a value, and will return a boolean result. Such predicates are associated with control-flow branches at design time, then evaluated at runtime to determine appropriate flow decisions based on the contextual costs of the current process instance.

The syntax of a cost-informed predicate is "cost(args) op numeric_value", where args may comprise:

- zero or one instance of case(), which may define within its parentheses a comma separated list of case identifiers and/or case identifier ranges (such as "250-300") and/or any one of the keywords: $\max$, min or average, and/or any 
of the keywords first, last or random followed by an integer (to select a block of cases), and/or either or both of the keywords from or to followed by a date (to filter on a date range), and/or the keyword dow followed by an integer (to filter on day of the week).

- zero or one instance of $\operatorname{task}()$, which may define within its parentheses a comma separated list of task identifiers.

- zero or one instance of resource(), which may define within its parentheses a comma separated list of resource identifiers.

All arguments act as filters over current and/or historical data. The identifiers and keywords listed in a case argument denote that the cost data returned will be limited to those cases. If none are listed inside the parentheses, then all previous and current cases of the current process model will be included. If the entire case argument is omitted from the cost predicate, then only the current case is considered.

Some examples of cost expressions:

- $\operatorname{cost}()$ returns the total cost of the current process instance up to the moment the predicate was evaluated

- $\operatorname{cost}(\operatorname{case}())$ returns the total cost of all past and current instances of the process

- $\operatorname{cost}($ case(average)) returns the average case cost of all past and current instances of the process

- cost(from 2014-01-01, dow 2, average) returns the average case cost of all process instances executed on any Monday since January 1, 2014.

- $\operatorname{cost}(\operatorname{case}(), \operatorname{task}(\mathrm{A}), \operatorname{resource}($ bloggsj)) returns the cost incurred by resource bloggsj performing task $\mathrm{A}$ in the current case

- cost(case(37, 62, 100-145, max), task(A, C, E), resource(bloggsj, 'Senior Manager')) returns the maximum cost incurred in a single case, of those cases listed, incurred by resource bloggsj or any member of the 'Senior Manager' role while performing the tasks A, C or E

Effect of Cost Predicates at Runtime. In YAWL, each outgoing branch of an OR- (or XOR-) split is associated at design time with a boolean predicate which, if evaluated to true at runtime, determines the branch (or branches, for a multi-OR split) that execution will follow. In this work, the YAWL workflow engine has been extended to accommodate control-flow predicates that include cost-based expressions. When process execution reaches such a predicate, the workflow engine will call the Cost Service via its evaluation interface, passing the expression, along with all associated data. The Cost Service will use that data, and any archival data as required, to evaluate the expression against the appropriate cost model components, and return the result. The engine will then embed the result into the predicate (replacing the cost-based expression), which it will then continue to evaluate in the usual manner, as needed. 
Resource Allocation. The YAWL Resource Service provides a pluggable framework for adding new resource allocation strategies, which at runtime receive the set of potential resources that may be allocated an activity, and use a defined strategy to select one resource from the set. The standard set of YAWL allocators (e.g. Random Choice, Shortest Queue, Round Robin) has been extended in this work with a number of cost-based strategies, such as Cheapest Resource, Cheapest to Start, Cheapest Completer and so on. When a cost-based allocator is enacted at runtime, it will directly query the Cost Service via either or both of its evaluation and log interfaces, and may request a calculation based on current and previous case histories (stored within the process logs) for the resources involved, based on its particular allocation strategy. The allocator will then use the result of the query to determine the appropriate resource to whom to allocate the activity, thus fulfilling a push-based resource interaction.

A user or administrator interacting with the YAWL worklist, which is also managed by the Resource Service, may use a button to invoke a query request to the Cost Service for cost data about a selected activity, which will then be displayed in a dialog. The user can then use this information to make ad-hoc cost-informed decisions regarding which activity to choose from their worklist to perform next, thus fulfilling a pull-based resource interaction (Fig. 2).

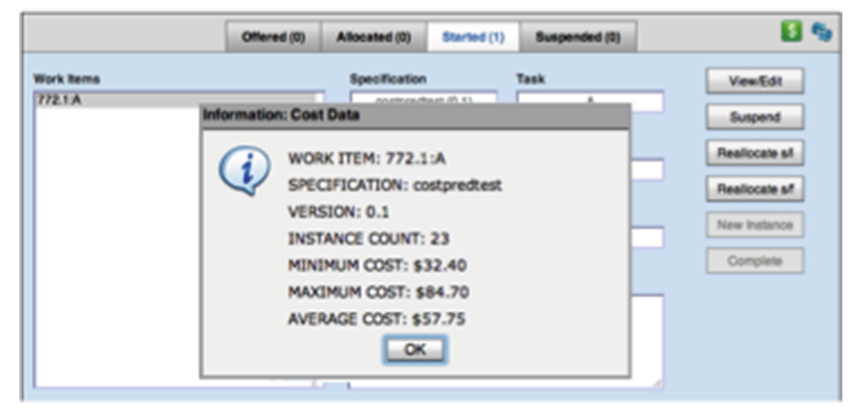

Fig. 2. The YAWL work list extended with cost information (example).

Process Variants. With regards to process variants, the standard YAWL environment includes a service called the Worklet Service that allows for the selection of process variants based on the current case context, available data values and an extensible rule set [3]. The rule semantics have been extended to accommodate the same cost-based expression syntax as that used for control-flow predicates. In addition, two comparison functions have been added, called cheapestVariant() and dearestVariant (), that will apply the associated cost expression against each variant available to a specified activity, and return the variant that is calculated as having the lowest or highest cost of the set, respectively.

When an activity is delegated to the Worklet Service, the Cost Service is queried for the calculated result of the associated rule from the rule set defined for that activity, which is then used to determine which process variant is the 
ideal selection based the current, cost-informed context of the case. For example, depending on the cost already expended on a certain case, the service may select a process variant that skips non-essential activities, or depending on the quoted costs from various suppliers of materials, select the variant designed to work directly with the systems of the chosen supplier.

\subsection{Cost Service Interaction Architecture}

Figure 3 shows the flow of information through the WfMS for each level of costinformed support.

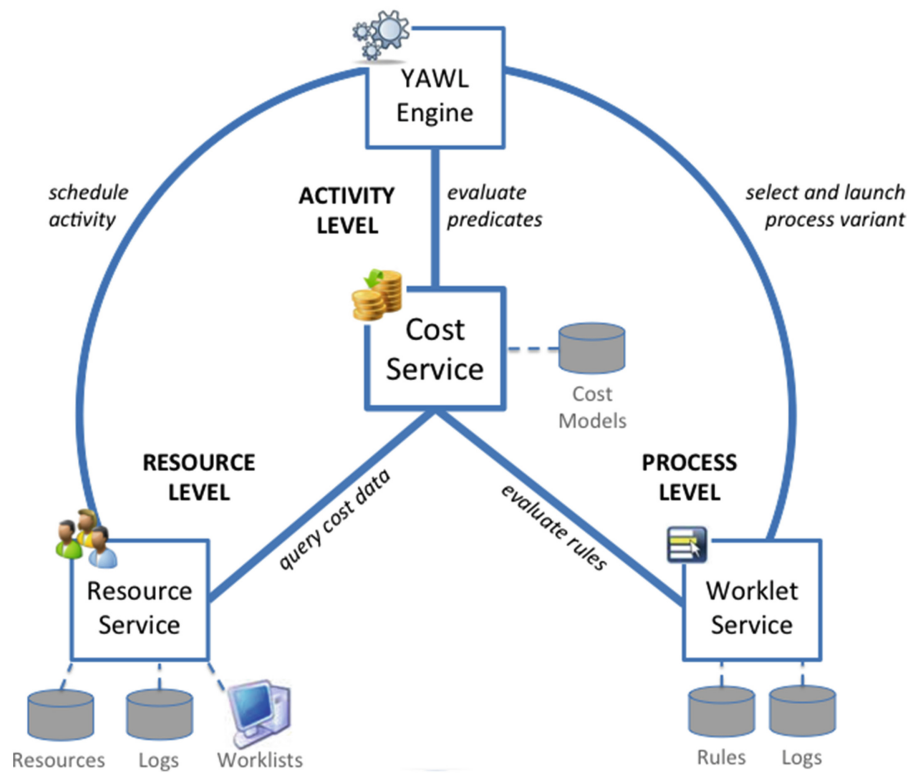

Fig. 3. Cost service interaction architecture.

At the process level, the Engine schedules an activity for execution by the Worklet Service, which traverses its rule set for the activity, querying the Cost Service to evaluate any cost expressions. The Cost Service evaluates and returns the result, which the Worklet Service uses to select the appropriate process variant for the activity, and launch the variant in the engine.

At the activity level, when the Engine encounters a branching construct in the control-flow of a process instance, it queries the Cost Service to evaluate any cost expressions in the predicate of each outgoing branch. The engine then uses the results of the predicate evaluations to fire the appropriate branch(es).

At the resource level, where the distribution of work takes place, the Engine schedules an activity with the Resource Service, which then queries the Cost Service for all cost information pertaining to the activity. If the activity is configured 
for system-based allocation (push pattern), the specified allocation strategy (e.g. Cheapest Resource) is employed using the cost information in its calculations, then the activity is routed to the worklist of the selected resource. If the activity is configured for resource-based allocation (pull pattern), the affected resources' worklists are updated with the retrieved cost information, allowing a resource to select the appropriate activity based on the cost information presented to them.

\section{$5 \quad$ Illustrative Example}

The YAWL process model depicted in Fig. 4, adapted from [7], represents a simplified home loan application and approval process that will serve as an example to illustrate the cost-informed support provided by the extensions to the YAWL environment. Tasks that require user interactions are annotated with role-based resource assignments. Most of the tasks in the process are assigned to one specific role, except for three parallel tasks in the (Re-)Assess Loan Application sub-process, which can be performed by a resource performing either the Mortgage Underwriter (MU) or Underwriting Assistant (UA) role. There are two tasks that do not require resourcing: Engage Broker will be delegated to the Worklet Service for execution of the appropriate variant at runtime, while Need Mortgage Insurance task will be executed internally by the YAWL engine using the information provided in the loan application.

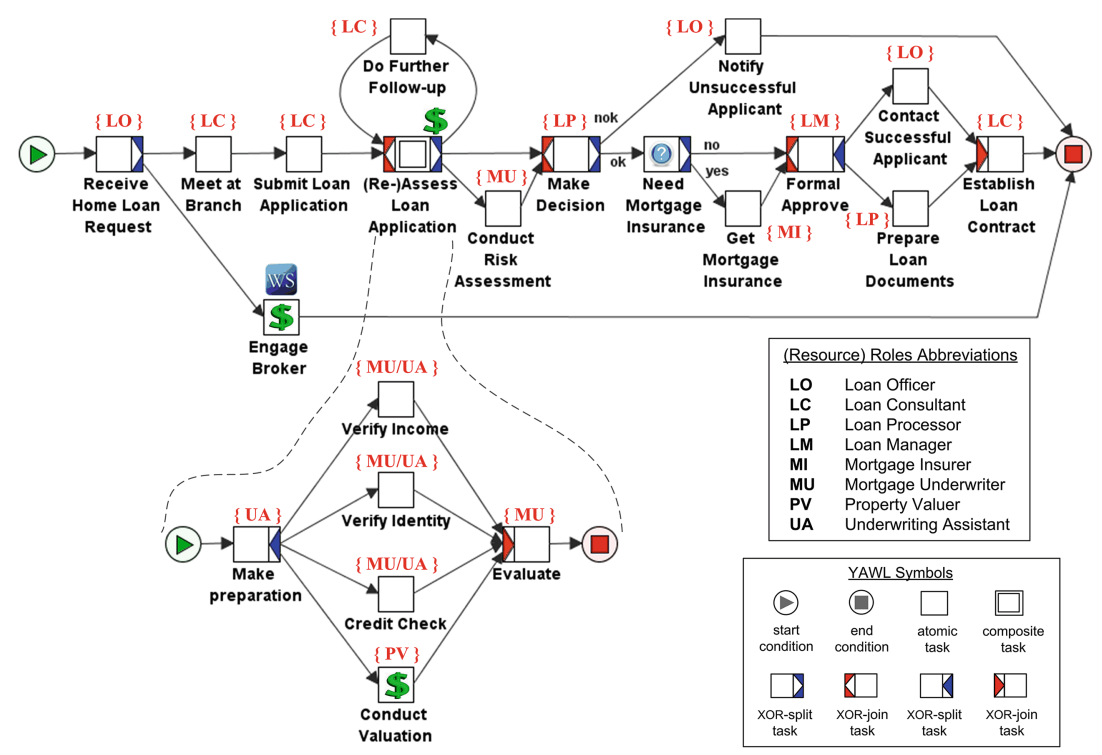

Fig. 4. A home loan process in YAWL (annotated with resource assignments).

The cost model for the process is populated based on the assumption that the cost optimisation strategy is to minimise the labour cost for processing a 
loan case wherever possible. The cost model includes the cost rates of resources and activities (modelled as tasks in the process model), of process variants (i.e. models supporting discrete mortgage broker services, managed by the Worklet Service), and of loan application cases (i.e. past and current instances of the process). The cost rates may be specified in different forms as follows:

- Role-based (variable or per hour) cost rate of a resource, e.g. a Loan Officer salary of $\$ 40$ per hour, or an Underwriting Assistant salary of $\$ 50$ per hour.

- Fixed cost rate of a resource for a given activity, e.g. to Conduct [Property] Valuation a senior Property Valuer charges $\$ 300$ while a junior Property Valuer charges $\$ 250$.

- Fixed cost rate of an activity, e.g. Get Mortgage Insurance incurs a mortgage insurance processing fee of $\$ 50$ per loan application (regardless of who conducts the activity).

- Variable cost rate of an activity, e.g. upon Formal Approval of a loan application a commission valued at $0.2 \%$ of the loan amount needs to be paid to the Loan Consultant who submitted the application.

- Variable cost rate of a process variant, e.g. a mortgage broker service provider charges a commission valued at $0.5 \%$ of the loan amount.

- Variable cost rate of a case, e.g. a disapproved loan application incurs an additional $\$ 100$ cost to the case, or an overdue case (of which the duration exceeds the service level agreement) receives a penalty of $\$ 150$ to the cost of the case.

The cost model is specified as an XML document and uploaded into the Cost Service for processing. The tasks annotated with $\$$ signs in the process model signify those activities where the Cost Service is called to evaluate a course of action during execution. Based on the above cost rates, cost rules are applied to support cost-informed decisions at each of the three levels during process execution.

Process Level. At runtime the Worklet Service handling the Engage Broker activity maintains a number of process variants corresponding to various broker services that charge the bank different commissions and/or fees for service provision. Cost-informed rules to guide the selection of a process variant may be based on the cost rate of service providers and/or their performance in previous cases (e.g. case duration). These rules are passed from the Worklet Service to the Cost Service for evaluation. For example, to select the variant that will engage with the broker service that has provided the cheapest average rate over the last 30 cases, the rule would be: cheapestVariant(case(average, last30)). Other contextual case data, such as loan amount, the ability to repay, the property location, and planning requirements, can also be used to further filter the result of the business rule.

Activity Level. The XOR-split following completion of the (Re-)Assess Loan Application activity is modelled to capture a normal business rule that may also embed a cost-based expression. In normal circumstances, either the Make 
Decision or the Do Further Follow-up activity will be enacted depending on the outcome of the application assessment. These rules are specified as flow predicates of the XOR-split. A flow predicate may also include a cost-informed expression that takes into account the process costs incurred, for example:

- If the (total) processing cost to this point (i.e. loan application assessment) reaches a certain limit (e.g. $0.5 \%$ of the loan amount), then instead of carrying out further follow-up, enact the Conduct Risk Assessment activity (expressed as $\operatorname{cost}() \geq($ loan_amt $* 0.005))$.

Resource level. Multiple resources assigned to perform the same activity may have different cost rates. Consider two typical examples: firstly, both Mortgage Underwriter and Underwriting Assistant can conduct the Credit Check activity but are paid a different hourly rate based on their roles; and secondly, even individual resources performing the same role may be paid at different salary levels. It is also likely that individuals performing the same role spend different amounts of time completing certain activities. All past histories of resource completion times for each activity across all process instances (of a particular model) are recorded in a process log, which may be used to define system-based allocation strategies for work distribution that select an available resource from the group of resources that perform the role. Examples are:

- When allocating a certain activity, select the available resource who offers the lowest overall cost rate from the role(s) designated to the activity (the Cheapest Resource allocation strategy). This strategy is used in the Conduct Valuation activity, where there are individual resources on different pay scales (junior and senior) performing the Property Valuer role.

- When allocating a certain activity, select the available resource who has historically completed the instances of the activity for the least cost, i.e. a function of cost rate and time taken to complete previous instances of the activity (the Cheapest Completer strategy). This strategy is used in the Evaluate activity, where there are three individual resources performing the Mortgage Underwriter role.

Figure 5 shows screen shots of design-time support for allocation strategy selection in the YAWL designer, while Fig. 6 shows screen shots of the organisational model, the related cost model, and the results of the system-based resource allocation during process execution in the YAWL runtime environment.

\section{Related Work}

Although cost efficiency is one of the key objectives of a business process management initiative $[8,9]$, there are only a limited number of research studies that examine in detail how cost measures are associated with business processes. The interrelationships between processes, resources and cost are well-known in the 


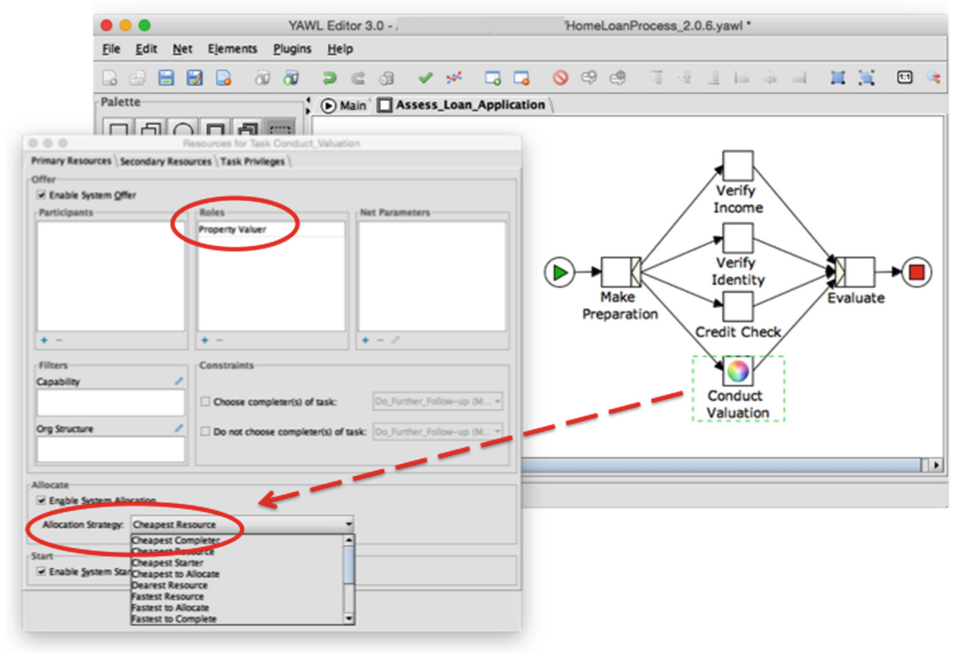

Fig. 5. At design time: the system is configured to allocate the "Conduct Valuation" activity to a resource with Property Valuer role with the cheapest cost.

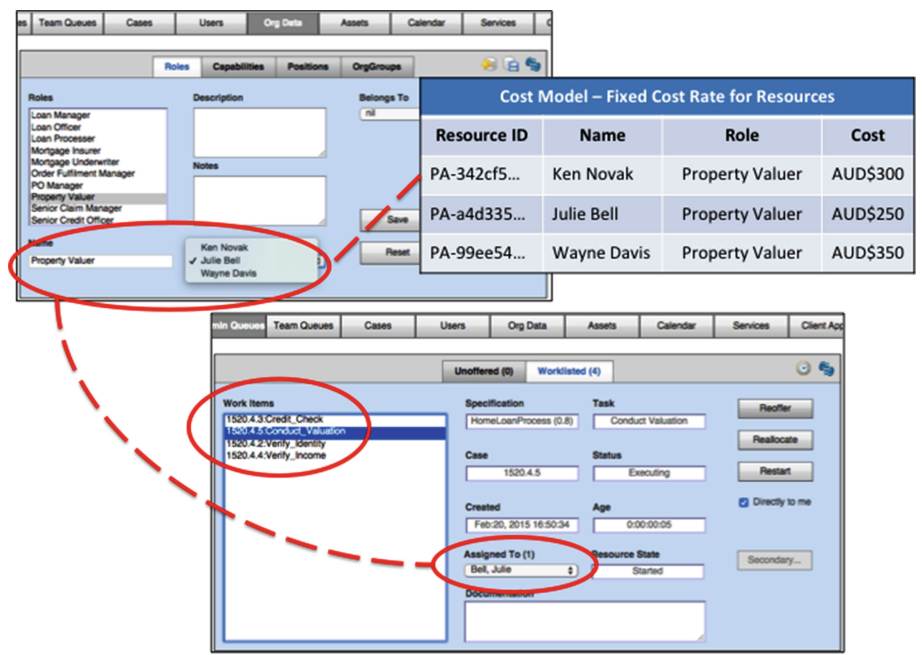

Fig. 6. At runtime: the system selects the resource with the cheapest cost (from the cost model) from all resources performing the "Property Valuer" role (from organisational data) and allocates the work item (instance of activity) "Conduct Valuation" to that resource.

accounting literature $[10,11]$ and several authors have studied the effects of ERP systems on accounting principles [12-14]. Recently, an information model to link the ARIS accounting structure with ARIS process semantics using Event Driven Process Chains (EPC) was proposed in [15] and an extensive set of cost measures to be linked to processes was proposed in [16]. 
Cost is typically considered as one of the dimensions for business process redesign and improvement [9,17]. It is also an important Quality of Services measure for web services [18-21] and is one of the trade-off considerations for grid and cloud computing [22-24].

Although WfMSs support planning, execution, (re)design and deployment of workflows [25], direct support for cost-informed execution is currently lacking. In our previous work, we presented a generic cost model for cost analysis using event logs $[4,26]$ and a framework to enable cost-informed operational process support [2]. In this paper, we demonstrated how such operational support based on cost considerations can be realised though an external cost service tightly coupled to the YAWL workflow management system.

\section{Conclusion and Future Work}

The paper proposes an architecture to provide cost-informed operational decision support for workflow management systems. The paper also presents a realisation of such a cost-informed workflow environment within the YAWL workflow management system in the form of a YAWL Cost Service (downloadable from www.yawlfoundation.org). In particular, we demonstrated using a running scenario how cost information can be linked to workflow specification as well as to resource specifications. Furthermore, we illustrated how cost-based decision rules for process variant selections, activity related decisions, and resource assignment decisions are all supported within the YAWL environment. By making use of the Cost Service, organisations can codify cost-based decision rules within a workflow management system and can monitor the cost of operations in real time. In terms of future work, we are interested in the development of techniques that can learn from these cost-informed operational decisions in order to improve process efficiency.

Acknowledgments. This work is supported by an ARC Discovery grant with number DP120101624. We would like to thank all our colleagues who were involved at various stages of this research study for their valuable input. They include Prof. Wil van der Aalst, Prof. Hajo Reijers, Prof. Michael Rosemann, Prof. Zahirul Hoque and Dr. Jochen De Weerdt.

\section{References}

1. Chung, L., Nixon, B., Yu, E., Mylopoulos, J.: Non-Functional Requirements in Software Engineering. Kluwer, Dordrecht (2000)

2. Wynn, M.T., Reijers, H.A., Adams, M., Ouyang, C., ter Hofstede, A.H.M., van der Aalst, W.M.P., Rosemann, M., Hoque, Z.: Cost-informed operational process support. In: Ng, W., Storey, V.C., Trujillo, J.C. (eds.) ER 2013. LNCS, vol. 8217, pp. 174-181. Springer, Heidelberg (2013)

3. ter Hofstede, A., van der Aalst, W., Adams, M., Russell, N. (eds.): Modern Business Process Automation: YAWL and Its Support Environment. Springer, Heidelberg (2010) 
4. Wynn, M.T., Low, W.Z., Nauta, W.: A framework for cost-aware process management: Generation of accurate and timely management accounting cost reports. In: Conferences in Research and Practice in Information Technology (CRPIT) (2013)

5. van der Aalst, W., Dustdar, S.: Process mining put into context. IEEE Internet Comput. 16, 82-86 (2012)

6. Russell, N., van der Aalst, W.M.P., ter Hofstede, A.H.M., Edmond, D.: Workflow resource patterns: identification, representation and tool support. In: Pastor, Ó., Falcão e Cunha, J. (eds.) CAiSE 2005. LNCS, vol. 3520, pp. 216-232. Springer, Heidelberg (2005)

7. Wynn, M., De Weerdt, J., ter Hofstede, A., Van Der Aalst, W., Reijers, H., Adams, M., Ouyang, C., Rosemann, M., Low, W.: Cost-aware business process management: a research agenda. In: 24th Australasian Conference on Information Systems (ACIS), pp. 1-11. RMIT University (2013)

8. Kettinger, W., Teng, J., Guha, S.: Business process change: a study of methodologies, techniques, and tools. MIS Q. 21(1), 55-80 (1997)

9. Reijers, H., Mansar, S.: Best practices in business process redesign: an overview and qualitative evaluation of successful redesign heuristics. Omega 33(4), 283-306 (2005)

10. Professional Accountants in Business Committee: Evaluating and improving costing in organizations, July 2009

11. Professional Accountants in Business Committee: Evaluating the costing journey: A costing levels continuum maturity model, July 2009

12. Booth, P., Matolcsy, Z., Wieder, B.: The impacts of enterprise resource planning systems on accounting practice-the Australian experience. Aust. Acc. Rev. 10(22), 4-18 (2000)

13. Grabski, S., Leech, S., Sangster, A.: Management Accounting in Enterprise Resource Planning Systems. CIMA Publishing, Oxford (2009)

14. Hyvönen, T.: Exploring Management Accounting Change in ERP Context. Ph.D. thesis. University of Tampere (2010)

15. vom Brocke, J., Sonnenberg, C., Baumoel, U.: Linking Accounting and ProcessAware Information Systems - Towards a Generalized Information Model for Process-Oriented Accounting. European Conference on Information Systems, pp. 1-13 (2011)

16. Sonnenberg, C., vom Brocke, J.: The missing link between BPM and accounting. Bus. Process Manage. J. 20, 213-246 (2014)

17. Netjes, M., Reijers, H.A., van der Aalst, W.M.P.: On the formal generation of process redesigns. In: Ardagna, D., Mecella, M., Yang, J. (eds.) Business Process Management Workshops. LNBIP, vol. 17, pp. 224-235. Springer, Heidelberg (2009)

18. Jaeger, M., Rojec-Goldmann, G., Muhl, G.: QoS aggregation for web service composition using workflow patterns. In: Eighth IEEE International Enterprise Distributed Object Computing Conference (EDOC 2004), pp. 149-159. IEEE (2004)

19. Cardoso, J., Sheth, A., Miller, J., Arnold, J., Kochut, K.: Quality of service for workflows and web service processes. Web Semant. Sci. Serv. Agents World Wide Web 1(3), 281-308 (2004)

20. Eckert, J., Repp, N., Schulte, S., Berbner, R., Steinmetz, R.: An approach for capacity planning for web service workflows. In: 13th Americas Conference on Information Systems (AMCIS 2007). Keystone, Colorado (2007)

21. Mohabbati, B., Gašević, D., Hatala, M., Asadi, M., Bagheri, E., Bošković, M.: A quality aggregation model for service-oriented software product lines based on variability and composition patterns. In: Kappel, G., Maamar, Z., Motahari-Nezhad, 
H.R. (eds.) Service Oriented Computing. LNCS, vol. 7084, pp. 436-451. Springer, Heidelberg (2011)

22. Yu, J., Buyya, R.: A budget constrained scheduling of workflow applications on utility grids using genetic algorithms. In: Workshop on Workflows in Support of Large-Scale Science, WORKS 2006, pp. 1-10, June 2006

23. Deelman, E., Singh, G., Livny, M., Berriman, B., Good, J.: The cost of doing science on the cloud: the Montage example. In: Proceedings of the 2008 ACM/IEEE Conference on Supercomputing, SC 2008, vol. 50, pp. 1-12. IEEE Press, Piscataway (2008)

24. Pandey, S., Barker, A., Gupta, K., Buyya, R.: Minimizing execution costs when using globally distributed cloud services. In: 2010 24th IEEE International Conference on Advanced Information Networking and Applications (AINA), pp. 222-229. IEEE (2010)

25. Weske, M.: Business Process Management: Concepts, Languages Architectures. Springer, New York (2007)

26. Wynn, M., Low, W., ter Hofstede, A., Nauta, W.: A framework for cost-aware process management: cost reporting and cost prediction. J. Univ. Comput. Sci. $\mathbf{2 0}(3), 406-430$ (2014) 